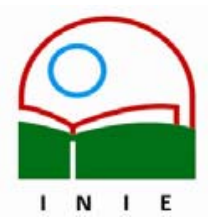

Universidad de Costa Rica

Facultad de Educación

Instituto de Investigación en Educación

ACTUALIDADES INVESTIGATIVAS EN EDUCACION

\title{
LA LECTURA EN LA ESCUELA COSTARRICENSE. ALGUNAS REFLEXIONES
}

\author{
Marielos Murillo Rojas ${ }^{1}$
}

\begin{abstract}
Resumen: El objetivo fundamental del presente artículo es analizar la concepción de lectura que subyace en los programas de español de l y II ciclos del Ministerio de Educación Pública (MEP) en Costa Rica y su adecuación al desarrollo del niño en cada una de las etapas de la escolaridad.

Se asume una concepción de lectura como proceso interactivo, en la que el lector es considerado como un sujeto activo que utiliza conocimientos de tipo muy variado para obtener información del texto y construir significados, de acuerdo con sus propios esquemas conceptuales abstraídos a partir del conocimiento del mundo.

Igualmente se revisan críticamente los lineamientos teórico-prácticos sobre la enseñanza de la lengua materna, según el MEP y específicamente se retoman los objetivos del área de lectura, cuyo núcleo de acción son unos niveles jerarquizados de comprensión de lectura, que se alejan de la concepción de lectura como proceso interactivo.

Para finalizar se describen algunos factores que favorecerian el proceso lector, por ejemplo; el distinguir entre situaciones de lectura de textos literarios y no literarios, experimentar diversidad de textos, enmarcar las actividades de lectura dentro de un ambiente afectivo, entre otras.
\end{abstract}

Palabras claves: LENGUA MATERNA/ LECTURA/ EDUCACIÓN PRIMARIA/ PROGRAMAS DE ESPAÑOL I Y II CICLOS DEL MEP/

\begin{abstract}
The central purpose of this paper is to analyse the concept of reading that underlies the teaching programs of the Spanish language and their adaptation programs to the development to the child in which one of the two cycles of the Costa Rican primary school as were approved by the Minister of Public Education (MEP).

It is supposed that the process of reading of any language is a process of interaction in which the reader plays an active role and uses all sort of knowledge to get information from the text and to integrate different meanings according to the conceptual models learned with her or his own experience on the world.

At the same time, the paper reviews critically the theoretic and practical foundations of the Spanish language teaching according to program officially approved by the MEO. Specifically it takes into account the objectives of the reading in which the nucleus of action is formed by some hierarchical levels of reading comprehension which contradicts the conception of reading as an interactive process.

Finally, the paper attempts to draw some factors that could facilitate the process of readership. For example, it tries to distinguish the process of reading literary texts from the reading of non literary texts, to experiment the use of different sorts of texts, to play the reading activities in an environment of affection, and so on.
\end{abstract}

Key words: MOTHER TONGUE / READING / PRIMARY SCHOOL EDUCATION / MINISTRY OF EDUCATION'S SPANISH PROGRAMS I \& II CYCLES

\footnotetext{
${ }^{1}$ Doctora en Formación del Profesorado con énfasis en didáctica de la lengua y la literatura española de la Universidad Extremadura, España. Máster en Lingüística y Licenciada en Educación Preescolar, ambos de la Universidad de Costa Rica. Profesora de la Escuela de Formación Docente de la misma Universidad. Áreas de interés la lectoescritura y el español como lengua materna en preescolar y primaria.
}

Correo electrónico: mamurillo2001cr@yahoo.com

Artículo recibido: 12 de julio, 2005

Aprobado: 22 de agosto, 2005 


\section{Introducción}

Es bien sabido que la enseñanza del lenguaje en la educación general básica debe propiciar el desarrollo de la expresión oral, de la comprensión oral, de la lectura y de la escritura, como elementos fundamentales para el desarrollo integral del niño y su correspondiente educación ligüística.

En consecuencia, al hablar de lectura y escritura estamos haciendo referencia en forma directa a la competencia lingüística-comunicativa del educando, a sus procesos de desarrollo, a su experiencia de vida, a su forma de interpretar el mundo y a sus necesidades e intereses.

La palabra -oral, escrita, expresada o comprendida- nos asegura la integración plena al mundo real o al mundo imaginario, siempre y cuando prevalezca ante todo la lectura de una realidad individual en proceso del desarrollo que, en el caso nuestro, debe ser favorecida por una intervención pedagógica efectiva y enriquecida con el contexto cultural en que se está inmerso.

Nuestra interrogante inicial ¿qué ha pasado con la lectura y la escritura en la escuela costarricense?, podría conducirnos hacia muchos caminos quizá inciertos; el Ministerio de Educación Pública (MEP) y sus políticas; el maestro y su formación académica y humana; la familia, como núcleo central de la sociedad y su co-responsabilidad en la formación del niño; los atractivos de la televisión; más recientemente los videojuegos; en fin, la lista aumenta según sigamos entretejiendo; pero para efectos de esta discusión me centraré únicamente en la escuela y el maestro.

La conceptualización de la lectura constituye el primer paso; para lo cual sigo a Colomer y Camps (2000), quienes, en su obra Enseñar a leer, enseñar a comprender, definen la lectura como la intelección de un texto y aseguran que

a pesar del reconocimiento espontáneo de la afirmación leer es entender un texto, la escuela contradice con cierta frecuencia esta afirmación al basar la enseñanza en una serie de actividades que se supone que mostrarán a los niños y niñas cómo se lee, pero en las que, paradójicamente, nunca es prioritario el deseo de que entiendan qué es lo que dice el texto. (Colomer y Camps, 2000, p. 33) 
Entre esas actividades se citan algunas muy comunes en el medio escolar costarricense, a saber: lectura de pequeños fragmentos textuales, palabras sueltas o en función de las letras que se estudian, letras aisladas según el orden de aparición preestablecido, lectura en voz alta centrada en la pronunciación correcta y la velocidad, todas consecuencia de una concepción de lectura que ha permanecido vigente durante siglos, pero que se aleja misma de lo que es la lectura.

La escuela costarricense, según mi opinión y la información que ofrecen los programas de español del Ministerio de Educación, I y II ciclos (1987/1990, 1991/1994, 1995/2000, 2001/2004 y 2005), ha estado casada por mucho tiempo con una concepción de lectura como procesamiento ascendente, en la que el lector inicia con los niveles inferiores del texto, para que llegue de forma sucesiva a los niveles superiores. No obstante, en los tres últimos programas se han dado algunas variaciones, por ejemplo el maestro tiene la libertad de elegir el método de lectoescritura deseado, pero en esencia se sigue concibiendo la lectura como un proceso jerarquizado y lineal; prueba de ello es el trabajo con niveles de lectura, lo que muestra un desconociendo acerca de la investigación realizada en los últimos tiempos y la lentitud del MEP para adecuarse a las demandas de las didácticas específicas, en este caso la lectura y a la ausencia de adecuación de los programas al desarrollo del niño a lo largo de la escolaridad.

Por otra parte, ¿habrán sido las universidades corresponsables de esta situación?, ¿se habrá dado las respuestas oportunas?, ¿se seguirán repitiendo prácticas que se alejan en mayor o menor medida de la labor comprometida que debe caracterizar el estudio de la didáctica de la lengua materna, específicamente en los procesos de: expresión oral, comprensión oral, lectura y escritura?

En lo que corresponde a la Universidad de Costa Rica, a partir de la década de 1990, se incrementan los estudios sobre la competencia comunicativa del educando (Programa ELEXHICÓS-INIL y algunas investigaciones del IIMEC, hoy INIE). Igualmente, el trabajo coordinado por profesores de la Escuela de Formación Docente con el Consejo Nacional de Lectura, de los esfuerzos realizados en los cursos específicos de las carreras de Educación Preescolar y de Educación Primaria, de las tesis de grado y seminarios de graduación relacionados con la competencia comunicativa de los educandos, de la incorporación del área del lenguaje en el proyecto de educación continua del Departamento de Primaria y 
Preescolar de la Escuela de Formación Docente, entre otras iniciativas. Pero todos estos esfuerzos no son suficientes; por tanto, urge iniciar una labor de coordinación con las instancias involucradas en la formación de los niños y jóvenes, con el propósito de establecer alianzas estratégicas y consensuar políticas claras que a mediano plazo permitan revertir la situación vivida hoy día, tal y como lo señala el propio Ministerio de Educación Pública: "La interacción verbal es sumamente importante para cualquier proceso educativo. Con frecuencia se afirma que una de las mayores causas de fracaso escolar es que los alumnos leen, escriben, se expresan oralmente y escuchan con notorias deficiencias" (MEP, 2005, p. 13)

\section{Concepto de lectura}

Destacar la importancia que han tenido la lectura y la escritura en el desarrollo de la humanidad y su valoración en todos los tiempos, puede parecer trivial en la medida en que formamos parte de una cultura letrada y en tanto la lectura y la escritura son parte indisociable de nuestro modus vivendi; pero como educadores debemos repetir y repetirnos a nosotros mismos que la lectura es y seguirá siendo una de las metas fundamentales de una educación con miras al desarrollo integral de la persona, y que es responsabilidad de todos, no solo del maestro de lengua y de literatura.

"La concepción actual de lectura se realiza en el interior del marco teórico general que explica cómo los seres humanos interpretan la realidad, cómo procesan la información". (Colomer y Camps, 2000, p. 34). En este sentido se habla de tres modelos sobre el proceso de lectura que han surgido a lo largo de las últimas décadas.

1- La lectura como proceso ascendente considera que, ante un texto, el lector procesa sus componentes empezando por las letras, las palabras, las frases, en un proceso ascendente que conduce a la comprensión del texto.

2- La lectura como proceso descendente parte de los conocimientos previos del lector estrato mental- y sobre esa base formula predicciones acerca del texto, para posteriormente buscar la información necesaria en ese texto, con el propósito de confirmar o refutar las predicciones hechas.

\footnotetext{
${ }^{2}$ El uso de negrita no está en el texto original.
} 
3- "Las dos formas de proceder, de abajo arriba y de arriba abajo, quedan englobadas en la idea básica de que, cuando una persona lee, parte de la hipótesis de que el texto posee un significado y lo que busca a través tanto del descubrimiento de indicios visuales como de la activación de una serie de mecanismos mentales que le permiten atribuirle un sentido, es decir, entenderlo. Lo que el lector ve en el texto y lo que él mismo aporta son dos subprocesos simultáneos y en estrecha interdependencia. Esta visión del proceso constituye lo que se llama modelos interactivos de lectura." (Colomer y Camps, 2000, p. 35)

En fin, en el modelo interactivo el lector es considerado como un sujeto activo que utiliza conocimientos de tipo muy variado para obtener información del texto y construir significado, de acuerdo con sus propios esquemas conceptuales conformados a partir del conocimiento del mundo.

Con el objetivo de ilustrar la forma en que el modelo interactivo de procesamiento paralelo utiliza todos los aspectos de los modelos ascendente y descendente, se cita el diagrama de Grace Williar (1999), tomado del Programa de comprensión de lectura en inglés, UNAM, México, 2003 (on line).

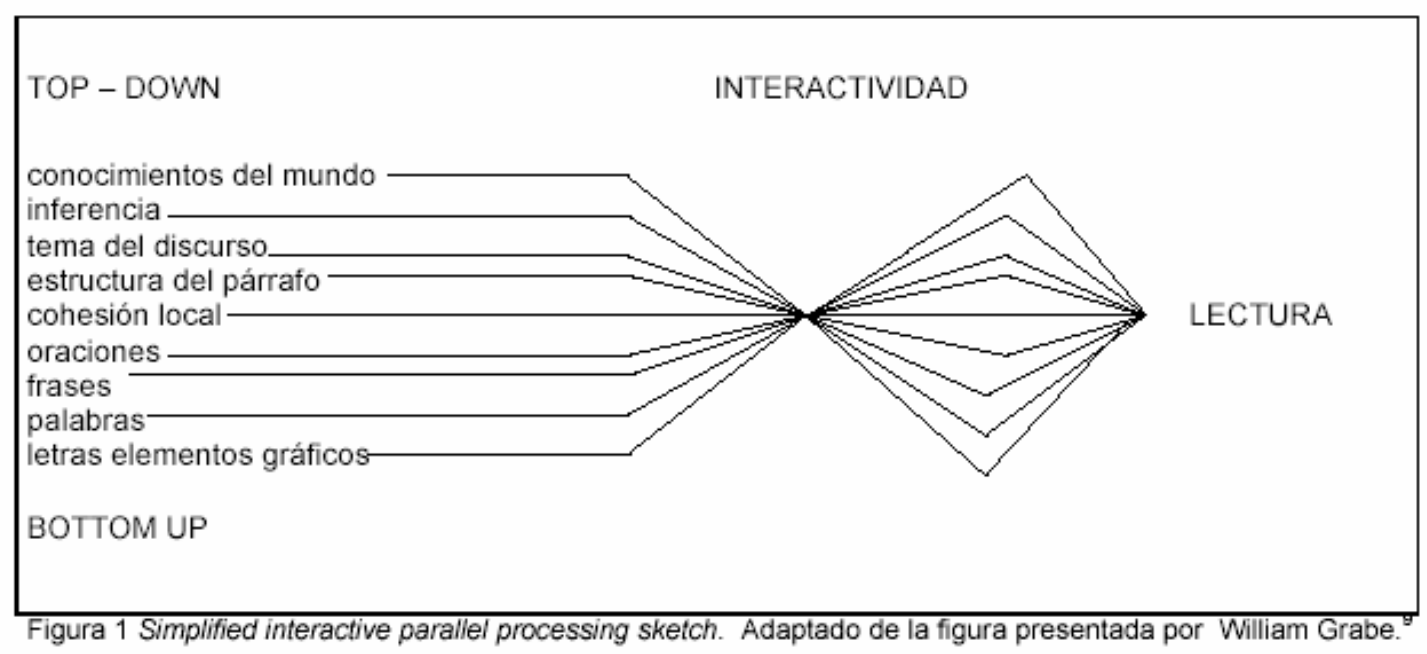

El orden de las fuentes de información citadas en el cuadro anterior, no implica una categorización determinada, ya que el lector las usa simultáneamente según sus necesidades. 


\section{Lineamientos teórico-prácticos sobre la enseñanza de la lengua materna, según el Ministerio de Educación Pública}

En el enfoque general de la enseñanza del español del Ministerio de Educación Pública, se reconoce que el adecuado dominio de las habilidades lingüístico-comunicativas constituye el cimiento de un proceso educativo exitoso, ya que tiene ingerencia directa en todas las otras áreas del currículum escolar.

Paralelamente se señala que el dominio deficiente de la lectura, escritura, expresión oral y comprensión oral constituye la causa fundamental del fracaso escolar.

Ante este panorama cabría preguntarse qué hace la escuela para revertir esa situación; es de esperar que los planteamientos teórico-prácticos de los programas del MEP den alguna luz, pero lamentablemente no es así, pues al leerlos cuidadosamente se encuentran contradicciones y reducciones teóricas que entran en juego y podrían confundir al lectormaestro que sigue al pie de la letra esos lineamientos programáticos, por ejemplo, la noción de aprestamiento inicia con la acertada recomendación de que se debe prestar especial atención a la estimulación lingüística del educando -motivación para la lectura, construcción del concepto de lectura y escritura, desarrollo de la expresión oral, estimulación del lenguaje escrito, aspectos perceptuales-; por tanto, es una labor de todo el proceso de escolarización, pero en párrafo siguiente se dice:

aunque el aprestamiento es una tarea a largo plazo, que corresponde a la educación preescolar, el maestro de primer grado debe realizar una revisión del desarrollo del niño en este campo para determinar las áreas deficitarias en sus estudiantes y elaborar un plan de atención según las necesidades del estudiante. (MEP, 2005, p. 14)

Como puede verse, en la práctica cotidiana este planteamiento reduccionista produce un efecto directo en el aula, los maestros de primer grado trabajan casi todo el primer trimestre del año escolar con procesos intensivos de aprestamiento, centrado en aspectos perceptuales y casi podría asegurar que dando énfasis al trabajo motriz.

Si se está de acuerdo con esta observación y esa es la realidad, tendríamos que preguntarnos por qué la escuela costarricense sigue desconociendo el trabajo realizado en 
preescolar y la experiencia acumulada por el niño como miembro de un mundo globalizado y de la información que lo enfrenta día a día con nuevos retos cognoscitivos.

El niño llega a la escuela con el afán de apropiarse de otras herramientas que le permitan integrarse plenamente al mundo como ser pensante, crítico y creativo y no a ejercitar destrezas motrices finas y gruesas, como lo señala en su primer objetivo el programa de español de primer grado.

Aquí radica el problema de base: nuestros niños llegan a primer grado con deseos de ser, hablar, leer y escribir, no de hacer bolitas, palitos, bodoquitos, etc.

Por otra parte, si según Rivers y Temperley (1978) y Gauquelin (1982), citados por Cassany 1997, respecto de la frecuencia de uso e importancia de las habilidades lingüísticas, el 45\% del tiempo lo dedicamos a escuchar, el $30 \%$ a hablar, el $16 \%$ a leer y el $9 \%$ a escribir, me pregunto por qué la escuela no promueve la escucha como proceso activo de comprensión, íntimamente ligado a la apropiación o aprehensión del mundo en sus más diversas manifestaciones culturales.

Escuchar es comprender mensajes varios, en contextos también varios, lo que permite al individuo ampliar sus horizontes, enriquecer sus esquemas mentales; en fin, ampliar su experiencia dándole mayor contenido a su forma de interpretar el mundo, requisito básico para la comprensión de otro tipo de texto, ya sea escrito o icónico.

Ahora bien, para tan importante habilidad lingüística el Ministerio de Educación sugiere "la práctica de actividades y técnicas que permitan discriminar los mensajes; reaccionar ante estímulos sensoriales; separar la idea principal del contexto y permanecer en silencio mientras otras personas hablan." (MEP, 2005, p. 15). Me pregunto dónde está en ese modelo de comprensión el contexto, el propósito, el tema de la situación de comunicación y el trabajo con los procesos de comprensión implícitos: reconocer, anticipar, inferir, retener, interpretar, seleccionar.

Pese a que la escucha es una de las habilidades lingüísticas que más practican los alumnos y que es uno de los procedimientos más relevantes para conseguir otros aprendizajes, no se trabaja de forma programada ni consciente en la escuela. 
Por otra parte, la expresión oral, hermana de la comprensión oral, también se ve relegada en el mejor de los casos a pequeños espacios, o sea, períodos de conversación o discusión sin ninguna articulación pedagógica, ya que no existe una propuesta clara que induzca al maestro de la educación general básica a contemplar dentro de la rutina diaria un espacio específico para la ejercitación de esta habilidad, o sea, un espacio para compartir un período de conversación, como lo tiene la educación preescolar.

Sin este espacio de escucha e interacción con los niños, surgen atinadamente las interrogantes de ¿cómo hace el maestro para saber qué está pasando con los procesos de desarrollo del alumno?, ¿cómo está procesando la información?, ¿qué papel está jugando su experiencia de vida?, o bien, ¿cómo asume el maestro su papel de mediador entre el conocimiento actual -viejo- y el nuevo que se está queriendo dar a conocer?, o sea, ¿cuál es su papel en esa zona de desarrollo próximo de la que habla Vigosky?

Por lo tanto, al ser la experiencia acumulada un elemento vital para construir el significado de un texto escrito, sin haber trabajado la expresión oral, difícilmente logremos éxito en la lectura, asumida como proceso de comprensión.

Respecto a la propuesta del Ministerio de Educación Pública sobre la lectura, se parte del hecho de que es un proceso activo de construcción de significados; sin embargo, se pliega a una concepción de comprensión de lectura en la que el lector realiza diferentes tipos de operaciones, organizados de manera ascendente, a saber: nivel literal, nivel inferencial, nivel de evaluación, nivel de apreciación, nivel de aplicabilidad y recreación.

Este planteamiento toma algunos de los componentes de la comprensión de lectura, considerados en las últimas investigaciones: literal, reorganizativo, inferencial y crítico (Catalá y otros, 2001, p. 16). Estos se agrupan básicamente para efectos de estudio y con el propósito de evidenciarlos al realizar las actividades de enseñanza y aprendizaje. No obstante, debe tenerse en cuenta que son simultáneos en el proceso lector $y$, por consiguiente, muchas veces inseparables y de ninguna forma deben ser la única guía orientadora de los procesos de lectura, sino más bien elementos por contemplar en la mediación pedagógica. 
En el ambiente escolar costarricense, según nuestra opinión y con base en los resultados desalentadores de las pruebas nacionales, considero que se han interpretado esos componentes como niveles jerarquizados y no como simultáneos en el proceso lector.

En vista de esta situación los objetivos específicos del área de lectura se redactan tomando como núcleo los niveles de comprensión de lectura presentes en el programa, por ejemplo:

\begin{tabular}{|c|c|}
\hline Objetivos & Contenidos \\
\hline $\begin{array}{l}\text { - Desarrollar las habilidades en la } \\
\text { comprensión lectora. (I Grado) }\end{array}$ & $\begin{array}{l}\text { Niveles de lectura (según lo especificado } \\
\text { en la fundamentación del programa) }\end{array}$ \\
\hline $\begin{array}{l}\text { - Aplicar los niveles de lectura para elevar } \\
\text { la comprensión de los textos. (II Grado) }\end{array}$ & $\begin{array}{l}\text { Lectura, según los diferentes niveles } \\
\text { especificados en la fundamentación de } \\
\text { estos programas (se debe escoger al } \\
\text { menos una obra acorde con este nivel, en } \\
\text { la bibliografía que se encuentra al final de } \\
\text { este programa). }\end{array}$ \\
\hline $\begin{array}{l}\text { - Aplicar los niveles de comprensión de } \\
\text { lectura. (II Grado) }\end{array}$ & $\begin{array}{l}\text { Secuencia de un relato. } \\
\text { Argumentos de cuentos. } \\
\text { Personajes principales y secundarios. }\end{array}$ \\
\hline $\begin{array}{l}\text { - Aplicar los niveles de comprensión } \\
\text { lectora. (III Grado) }\end{array}$ & $\begin{array}{l}\text { Niveles de comprensión de lectura. } \\
\text { La descripción. } \\
\text { Lectura según el modelo propuesto en la } \\
\text { fundamentación de este programa. }\end{array}$ \\
\hline $\begin{array}{l}\text { - Aplicar en descripciones y narraciones, } \\
\text { los diferentes niveles de la comprensión } \\
\text { lectora, según la fundamentación de este } \\
\text { programa. (IV Grado) }\end{array}$ & $\begin{array}{l}\text { La descripción. } \\
\text { Niveles de comprensión de lectura. } \\
\text { Figuras literarias: personificación, símil y } \\
\text { metáfora. } \\
\text { La narración. }\end{array}$ \\
\hline
\end{tabular}




\begin{tabular}{|l|l|}
\hline $\begin{array}{l}\text { - Aplicar niveles de lectura a textos } \\
\text { literarios y no literarios. (V Grado) }\end{array}$ & $\begin{array}{l}\text { Niveles de lectura en textos literarios y no } \\
\text { literarios. }\end{array}$ \\
\hline $\begin{array}{l}\text { - Aplicar niveles de lectura a textos de } \\
\text { mediana extensión y complejidad para } \\
\text { mejorar la comprensión. (VI Grado). }\end{array}$ & Niveles de lectura. \\
\hline
\end{tabular}

Como puede observarse en el cuadro anterior, leer es casi sinónimo de la apropiación de los diferentes niveles de comprensión, obviando claramente los otros aspectos que entran en juego en un proceso interactivo de lectura, tal y como fue descrito en el punto anterior.

Por otra parte, ese planteamiento oficial desconoce parcialmente los verdaderos factores que condicionan la comprensión de lectura, o sea, el lector y el texto, pues no son tomados en cuenta en su totalidad.

Desde el punto de vista del lector debe considerarse: la intención de la lectura y los conocimientos previos.

La intención de la lectura determinará la forma en que el lector abordará el escrito y el nivel de comprensión que asumirá para dar por buena su lectura. "No es lo mismo, por ejemplo, leer para retener una información, para aprender y reestructurar conocimientos, que leer para formarse la idea general, para saber de qué va un libro." (Colomer y Camps, 2000, p. 55). Igualmente el lector no se acerca con la misma intención a una narración, un libro de cocina, una revista de entretenimiento, una enciclopedia, un diccionario, un libro de texto, entre otros.

Respecto de los conocimientos aportados por el lector, este debe poseer información y vivencias de tipo muy variado para poder abordar con éxito su lectura. En consecuencia, es preciso conocer la situación comunicativa, qué objetivo tiene, desde qué lugar y tiempo se produce; características textuales y formales del texto escrito; conocimientos previos, o sea, la información que el lector posee sobre los acontecimientos de que trata la lectura, ya que el 
grado de conocimiento compartido entre el emisor y el receptor asegura la comprensión del texto.

En suma, "la comprensión del texto resulta muy determinada por su capacidad de escoger y de activar todos los esquemas de conocimiento pertinentes para un texto concreto." (Colomer y Camps, 2000, p. 56)

\section{Factores de éxito que favorecen el proceso lector}

Pensar, hablar, escuchar, escribir y leer son partes diferentes de un mismo acto, que tiene sentido cuando tiene una finalidad: la comunicación. Es por este motivo que no podemos separar la lectura y hacer de ella algo independiente, sino que la deberíamos unir a las producciones escritas por los niños y niñas, en un ambiente familiar y distendido, donde el niño se sienta atendido y querido. (Catalá y otras, 2001, p. 21)

Las condiciones que favorecen el aprendizaje lector son: el ambiente afectivo en que se enmarquen las actividades de lectura y escritura, el gusto y disfrute que se proyecte durante la lectura animada (aquella capaz de atraer la atención del receptor y de permitirle construir significados), la conceptualización de la lectura solo como un medio para adquirir nuevos aprendizajes y desarrollar la imaginación.

Bajo este enfoque es fundamental distinguir entre la lectura de textos literarios y la de textos no literarios. Ambas, complementos de un mismo proceso.

Al respecto, es menester escuchar lo que dicen los mismos niños costarricenses, por ejemplo en una encuesta sobre lectura, realizada por Ibarra y otras, 1996, los escolares manifestaron que la lectura y la escritura son las actividades escolares que les generan menos agrado y como consecuencia no visitan librerías ni bibliotecas, a no ser que deban realizar tareas obligatorias; unido a esta penosa situación externaron su disgusto por los libros que están en la escuela, ya que no responden a sus intereses inmediatos.

En esa misma investigación se afirma que los niños no poseen habilidades y destrezas de comprensión de lectura, debido a un inadecuado proceso metodológico que utilizan las docentes para la enseñanza, a la poca colaboración de las familias, al costo elevado de 
libros, a los materiales poco atractivos que ofrecen las bibliotecas escolares y comunales y, en general, a la ausencia de una cultura que promueva la lectura como medio de comunicación.

En fin,

para conseguir buenos lectores, capaces para asimilar la información que les proporciona la lectura y hacer un uso creativo de ella, es esencial la actitud emocional que se cree ante la misma. Por eso, el niño ha de sentir que la lectura forma parte de la vida, que es un medio extraordinario de comunicación y de información. Esta experiencia cotidiana y vivida a lo largo de su paso por la escuela es la que dará sentido a este esfuerzo de asimilación. (Catalá y otras, 2001, p. 21)

Siguiendo con la concepción de lectura, compartida por Colomer y Camps, 2000, las condiciones básicas para la enseñanza de la lectura serían las siguientes:

- Partir de lo que los alumnos saben: conocimiento del mundo.

- Favorecer la comunicación descontextualizada: interrelacionar la lengua escrita con el dominio de las otras habilidades lingüísticas.

- Familiarizar a los alumnos con la lengua escrita y crear una relación positiva con el escrito: conciencia del uso funcional de la escritura, con el propósito de alejar la lectura con el ámbito eminentemente escolar.

- Fomentar la conciencia metalingüística: estudio del lenguaje como objeto en sí mismo.

- Utilizar textos concebidos para la lectura: evitar textos artificiales y simplificados.

- Experimentar la diversidad de textos y lecturas: desarrollo de las habilidades necesarias para proceder conforme los diferentes tipos de textos.

- Leer sin tener que oralizar: Centrarse en la atención en la construcción del sentido y no en el descifrado.

- La lectura en voz alta: entendida como una situación de la comunicación oral en la que alguien desea transmitir lo que dice el texto y no una simple oralización como respuesta a la solicitud del adulto. 


\section{A manera de conclusión}

1. La formación teórico-práctica del docente debe focalizar en su trabajo, en forma integral y consciente, las habilidades lingüísticas, en el presente caso la lectura.

2. Las universidades, como centros difusores de cultura, deben comprometerse con programas de animación a la lectura, círculos de lectura y discusión de problemas que atañan a las didácticas del contenido, mediante estrategias de educación continua y de apoyo directo al Ministerio de Educación.

3. La organización del trabajo escolar debe fomentar las capacidades de los alumnos y sus responsabilidades, de tal forma que aquellos exterioricen qué quieren saber y cómo quieren aprenderlo, qué tipo de textos se acercan más a sus intereses. De atenderse las necesidades de los educandos, con seguridad la lectura creará espacios significativos de crecimiento cultural.

4. Es conveniente que el Ministerio de Educación Pública en coordinación con las Universidades Estatales rediseñe los programas de Lengua Materna, a la luz de las bases científicas de la didáctica de la lengua y la literatura, a saber: marco discusivo y sociocultural, aportes de la teoría lingüística - pragmática, semiótica, lingüística del texto y análisis del discurso-, teoría literaria y enfoque psicopedagógico.

\section{Referencias}

Benavides, Jennory y otras. (1996). Competencia en la comprensión de la Lectura de escolares que finalizan la Educación General Básica. Seminario de Graduación, para optar por el título de licenciatura en Educación Primaria. Universidad de Costa Rica.

Cassany, Daniel y otras. (1997). Enseñar lengua. Barcelona: Graó.

Catalá, Gloria y otras. (2001). Evaluación de la comprensión lectora. Barcelona: Graó.

Colomer, T. y Camps, A. (1996/2000). Enseñar a leer, enseñar a comprender. Madrid: Celeste/M.E.C. 
Ministerio de Educación Pública-C.R. (1987). Contenidos básicos para el I y II ciclo de la Educación General Básica. San José: Departamento de publicaciones Ministerio de Educación Pública.

Ministerio de Educación Pública-C.R. (1991). Programa de estudios. Español I y II ciclo de la Educación General Básica. San José: Departamento de publicaciones Ministerio de Educación Pública.

Ministerio de Educación Pública-C.R. (1995). Programa de estudios. Español I y II ciclo de la Educación General Básica. San José: Departamento de publicaciones Ministerio de Educación Pública.

Ministerio de Educación Pública-C.R. (2005). Programa de estudios. Español I y II ciclo de la Educación General Básica. San José: Departamento de publicaciones Ministerio de Educación Pública.

Universidad Nacional Autónoma de México, Colegio de ciencias y humanidades. (2003). Suplemento de los programas de comprensión de lectura en Inglés, I, II, III y IV. Recuperado el junio del 2005, de www.cch.unam.mx/planesyprogramas/programasajustados/inglés/suplemento/PDF 